\title{
ON HOMOGENEOUS SPACES AND REDUCTIVE SUBALGEBRAS OF SIMPLE LIE ALGEBRAS
}

\author{
BY \\ A. SAGLE AND D. J. WINTER( $\left.{ }^{(}\right)$
}

1. Introduction. Let $G$ be a connected Lie group and $H$ a closed subgroup. Then the homogeneous space $M=G / H$ is called reductive if in the Lie algebra $g$ of $G$ there exists a subspace $\mathfrak{m}$ such that $\mathfrak{g}=\mathfrak{m}+\mathfrak{h}$ (subspace direct sum) and $[\mathfrak{h}, \mathfrak{m}]$ $\subset \mathfrak{m}$ where $\mathfrak{h}$ is the Lie algebra of $H$ (see [4], [5]). In this case the pair $(\mathfrak{g}, \mathfrak{h})$ is called a reductive pair and the subspace $\mathfrak{m}$ can be made into an anti-commutative algebra as follows. For $X, Y \in \mathfrak{m}$ let $[X, Y]=X Y+\mathfrak{h}(X, Y)$ where $X Y=[X, Y]_{\mathfrak{m}}$ (resp. $\left.\mathfrak{h}(X, Y)=[X, Y]_{\mathfrak{h}}\right)$ is the projection of $[X, Y]$ in $\mathfrak{g}$ into $\mathfrak{m}$ (resp. $\left.\mathfrak{h}\right)$. This algebra is related to the canonical $G$-invariant connection $\nabla$ of the first kind on $G / H$ by $\left[\nabla_{X^{*}}\left(Y^{*}\right)\right]_{P_{0}}=\frac{1}{2} X Y$ where $P_{0}=H \in M$ (see [5, Theorem 10.1]).

For a fixed decomposition $\mathfrak{g}=\mathfrak{m}+\mathfrak{h}$, the Lie algebra identities of $\mathfrak{g}$ yield the following identities for $\mathfrak{m}$ and $\mathfrak{h}$. For $X, Y, Z \in \mathfrak{m}$ and $U \in \mathfrak{h}$,

(1) $X Y=-Y X$ (bilinear);

(2) $\mathfrak{h}(X, Y)=-\mathfrak{h}(Y, X) \quad$ (bilinear);

(3) $[Z, \mathfrak{h}(X, Y)]+[X, \mathfrak{h}(Y, Z)]+[Y, \mathfrak{h}(Z, X)]=J(X, Y, Z) \equiv(X Y) Z+(Y Z) X$ $+(Z X) Y$.

(4) $\mathfrak{h}(X Y, Z)+\mathfrak{h}(Y Z, X)+\mathfrak{h}(Z X, Y)=0$;

(5) $\mathfrak{h}[(X, Y), U]=\mathfrak{h}([X, U], Y)+\mathfrak{h}(X,[Y, U])$;

(6) $[U, X Y]=[U, X] Y+X[U, Y]$.

In particular (6) says the mappings $\operatorname{ad}_{\mathfrak{m}} U: \mathfrak{m} \rightarrow \mathfrak{m}: X \rightarrow[U, X]$ are derivations of the algebra $\mathrm{m}$. Using these identities, there was established in [6] a correspondence between simple algebras $\mathfrak{m}$ and holonomy irreducible simply connected spaces $G / H$ which are not symmetric $(\mathfrak{m m}=0$ if and only if $G / H$ is a symmetric space); for example, if $G / H$ is riemannian, then $G / H$ is holonomy irreducible if and only if $\mathfrak{m}$ is a simple algebra.

In this paper, we consider pairs $(\mathfrak{g}, \mathfrak{h})$ where $g$ is a simple Lie algebra over a field $F$ of characteristic zero and $\mathfrak{h}$ is either semisimple, or regular and reductive (see [2]). In each case we show that the associated $\mathfrak{m}$ is either simple or abelian $\left(\mathfrak{m}^{2}=0\right)$. This together with [6] shows in particular that if $G$ is a simple connected Lie group and $H$ a closed semisimple or regular reductive Lie subgroup of $G$ such that $G / H$ is simply connected, then either $G / H$ is a symmetric space or $G / H$ is holonomy irreducible. This is a reasonable account of the situation since it can be shown that

Received by the editors July 19, 1966.

( ${ }^{1}$ Research supported by NSF Grant GP-1453 and by Army Research Office, Durham (respectively). 
if $G / H$ is a holonomy irreducible pseudo-riemannian reductive space with $G$ simple, then $\mathfrak{h}$ is a reductive subalgebra of $\mathfrak{g}$.

\section{The regular reductive case.}

LEMMA 1. Let $\mathfrak{a}$ be a nonassociative algebra with derivation algebra Der a. Assume that $\mathfrak{a}$ has no proper ideal stable under Der $\mathfrak{a}$. Then either $\mathfrak{a}$ is simple or $\mathfrak{a}^{2}=0$.

Proof. Assume $\mathfrak{a}^{2} \neq 0$ and let $\mathfrak{T}(\mathfrak{a})$ denote the associative algebra generated by the left and right multiplications of $\mathfrak{a}[3$, p. 290]. Let $R$ be the radical of $\mathfrak{I}(\mathfrak{a})$. Then $R \mathfrak{a}$ is an ideal of $\mathfrak{a}$ since $\mathfrak{T}(\mathfrak{a})(R \mathfrak{a}) \subseteq(\mathfrak{T}(\mathfrak{a}) R) \mathfrak{a} \subseteq R \mathfrak{a}$. If $D \in \operatorname{Der} \mathfrak{a}$, then $[D, \mathfrak{T}(\mathfrak{a})]$ $\subseteq \mathfrak{T}(\mathfrak{a})$ since $\operatorname{ad}_{\text {Hom(a,a) }} D$ stabilizes the set of right and left multiplications (e.g., $[D, L(A)]=L(D(A))$ where $L(B)$ denotes left multiplication by $B$ in a). Thus $\operatorname{ad}_{\mathfrak{T}(a)} D$ is a derivation of $\mathfrak{T}(\mathfrak{a})$ and it follows that $[D, R] \subseteq R[3$, p. 30, exercise 22]. Thus $D(R \mathfrak{a}) \subseteq[D, R] \mathfrak{a}+R(D \mathfrak{a}) \subseteq R \mathfrak{a}$. Thus $R \mathfrak{a}$ is a Der $\mathfrak{a}$-stable ideal of $\mathfrak{a}$. By assumption, we must have $R \mathfrak{a}=\mathfrak{a}$ or $R \mathfrak{a}=0$. If $R \mathfrak{a}=\mathfrak{a}$, then for some $i$, $0=R^{\mathfrak{i}} \mathfrak{a}=R^{i-1} \mathfrak{a}=\cdots=R \mathfrak{a}=\mathfrak{a}$ and $\mathfrak{a}=0$. Thus we may assume that $R \mathfrak{a}=0$. Then $R=0$ and $\mathfrak{I}(\mathfrak{a})$ is completely reducible on $\mathfrak{a} . \mathfrak{a}^{2}$ is clearly Der $\mathfrak{a}$-stable. Assuming that $\mathfrak{a}^{2} \neq 0$, we must have $\mathfrak{a}^{2}=\mathfrak{a}$ by hypothesis. We claim that $\mathfrak{a}^{2}=\mathfrak{a}$ implies that $\mathfrak{a}$ is simple. For if $\mathfrak{b}$ were a proper ideal of $\mathfrak{a}$, then $\mathfrak{b}$ would be $\mathfrak{T}(\mathfrak{a})$-stable and hence $\mathfrak{a}=\mathfrak{b} \oplus \mathfrak{b}^{\prime}$ for some $\mathfrak{T}(\mathfrak{a})$-stable $\mathfrak{b}^{\prime}$. This $\mathfrak{b}^{\prime}$ would be an ideal and $\mathfrak{a}=\mathfrak{a}^{2}=\mathfrak{b}^{2}+\left(\mathfrak{b}^{\prime}\right)^{2}$ shows that $\mathfrak{b}^{2}=\mathfrak{b}$. But then $\mathfrak{b}=\mathfrak{b}^{2}$ would be Der $\mathfrak{a}$-stable since for $B_{1}, B_{2}$ in $\mathfrak{b}$, $D\left(B_{1} B_{2}\right)=\left(D B_{1}\right) B_{2}+B_{1}\left(D B_{2}\right) \in \mathfrak{b}$. Thus $\mathfrak{a}$ is simple.

We now consider reductive pairs $(\mathfrak{g}, \mathfrak{h})$. Thus let $\mathfrak{g}$ be a Lie algebra, $\mathfrak{h}$ a Lie subalgebra of $\mathfrak{g}, \mathfrak{m}$ a complementary subspace of $\mathfrak{h}$ in $\mathfrak{g}$ such that $[\mathfrak{m} \mathfrak{h}] \subseteq \mathfrak{m}$. For $X, Y \in \mathfrak{m}$ we define $X Y$ in $\mathfrak{m}$ and $\mathfrak{h}(X, Y)$ in $\mathfrak{h}$ by requiring that $[X Y]=X Y$ $+\mathfrak{h}(X, Y)$. We regard $\mathfrak{m}$ as a nonassociative algebra with respect to the product $X Y$. Then $\mathfrak{m}$ is clearly anti-commutative and $\operatorname{ad}_{\mathfrak{m}} U$ is a derivation of $\mathfrak{m}$ for $U \in \mathfrak{h}$ (by (6)).

LEMMA 2. Let $\mathfrak{n}$ be an ad $\mathfrak{h}$-stable ideal of $\mathfrak{m}$. Let $\mathfrak{q}=\mathfrak{n}+\mathfrak{h}(\mathfrak{n}, \mathfrak{n})$. If $\left[\mathfrak{n}, \mathfrak{n}^{\prime}\right] \subseteq \mathfrak{q}$ for some complementary subspace $\mathfrak{n}^{\prime}$ of $\mathfrak{n}$ in $\mathfrak{m}$, then $\mathfrak{q}$ is an ideal of $\mathfrak{g}$.

Proof. $[\mathfrak{q}, \mathfrak{n}] \subseteq[\mathfrak{n}, \mathfrak{n}]+[\mathfrak{h}(\mathfrak{n}, \mathfrak{n}), \mathfrak{n}] \subseteq \mathfrak{n} \mathfrak{n}+\mathfrak{h}(\mathfrak{n}, \mathfrak{n})+\mathfrak{n}$ by (3) since $\mathfrak{n}$ is ad $\mathfrak{h}$-stable. Thus $[\mathfrak{q}, \mathfrak{n}] \subseteq \mathfrak{q}$. And $[\mathfrak{q}, \mathfrak{h}] \subseteq \mathfrak{q}$ since $\mathfrak{n}$ is ad $\mathfrak{h}$-stable and $\mathfrak{q}=\mathfrak{n}+[\mathfrak{n}, \mathfrak{n}]$. It remains to show that $\left[\mathfrak{q}, \mathfrak{n}^{\prime}\right] \subseteq \mathfrak{q}$. But we have

$$
\begin{aligned}
{\left[\mathfrak{q}, \mathfrak{n}^{\prime}\right] } & \subseteq \mathfrak{n} \mathfrak{n}^{\prime}+\mathfrak{h}\left(\mathfrak{n}, \mathfrak{n}^{\prime}\right)+\left[\mathfrak{h}(\mathfrak{n}, \mathfrak{n}), \mathfrak{n}^{\prime}\right], \\
{\left[\mathfrak{h}(\mathfrak{n}, \mathfrak{n}), \mathfrak{n}^{\prime}\right] } & \subseteq\left[\mathfrak{n} \mathfrak{n}, \mathfrak{n}^{\prime}\right]+\left[[\mathfrak{n}, \mathfrak{n}], \mathfrak{n}^{\prime}\right] \subseteq\left[\mathfrak{n}, \mathfrak{n}^{\prime}\right]+\left[\mathfrak{n},\left[\mathfrak{n}, \mathfrak{n}^{\prime}\right]\right], \\
\mathfrak{h}\left(\mathfrak{n}, \mathfrak{n}^{\prime}\right) & \subseteq \mathfrak{n} \mathfrak{n}^{\prime}+\left[\mathfrak{n}, \mathfrak{n}^{\prime}\right] .
\end{aligned}
$$

But since $\left[\mathfrak{n}, \mathfrak{n}^{\prime}\right] \subseteq \mathfrak{q}$ by hypothesis, $\mathfrak{q}$ contains $\left[\mathfrak{h}(\mathfrak{n}, \mathfrak{n}), \mathfrak{n}^{\prime}\right]$ (using (3)) and $\mathfrak{h}\left(\mathfrak{n}, \mathfrak{n}^{\prime}\right)$. Since $\mathfrak{n} \mathfrak{n}^{\prime} \subseteq \mathfrak{n}(\mathfrak{n}$ is an ideal of $\mathfrak{m}),\left[\mathfrak{q}, \mathfrak{n}^{\prime}\right] \subseteq \mathfrak{q}$. Thus $\mathfrak{q}$ is an ideal of $\mathfrak{g}$.

Lemma 3. Suppose that the Killing form $B(, \quad)$ of $\mathfrak{g}$ is nondegenerate and that $B(\mathfrak{m}, \mathfrak{h})=0$. Then $B() \mid, \mathfrak{m}$ is nondegenerate and invariant, i.e., $B(X Y, Z)$ $=B(X, Y Z)$. Moreover every ad $\mathfrak{h}$-stable ideal $\mathfrak{n}$ of $\mathfrak{m}$ satisfies $\left[\mathfrak{n}, \mathfrak{n}^{\perp}\right]=0$ where $\mathfrak{n}^{\perp}=\{X \in \mathfrak{m} \mid B(X, \mathfrak{n})=0\}$. 
Proof. For $X, Y, Z \in \mathfrak{m}$ we have:

$$
\begin{aligned}
B(X Y, Z) & =B([X, Y]-\mathfrak{h}(X, Y), Z)=B([X, Y], Z)=B(X,[Y, Z]) \\
& =B(X, Y Z+\mathfrak{h}(Y, Z))=B(X, Y Z) .
\end{aligned}
$$

Now $B\left(\mathfrak{n}^{\perp}, \mathfrak{n}\right)=0$ implies that $0=B\left(\mathfrak{n}^{\perp}, \mathfrak{n m}\right)=B\left(\mathfrak{n} \mathfrak{n}^{\perp}, \mathfrak{m}\right)$. And $B(\mathfrak{m}, \mathfrak{h})=0$ implies that $B\left(\mathfrak{n} \mathfrak{n}^{\perp}, \mathfrak{h}\right)=0$. Thus $B\left(\mathfrak{n} \mathfrak{n}^{\perp}, \mathfrak{g}\right)=0$ and $\mathfrak{n} \mathfrak{n}^{\perp}=0$. Consequently $\left[\mathfrak{n}, \mathfrak{n}^{\perp}\right]=\mathfrak{h}\left(\mathfrak{n}, \mathfrak{n}^{\perp}\right)$ $\subseteq \mathfrak{h}$ and $B\left(\left[\mathfrak{n}, \mathfrak{n}^{\perp}\right], \mathfrak{m}\right)=0$. But we also have $B\left(\left[\mathfrak{n}, \mathfrak{n}^{\perp}\right], \mathfrak{h}\right)=B\left(\mathfrak{n}^{\perp},[\mathfrak{n} \mathfrak{h}]\right)=B\left(\mathfrak{n}^{\perp}, \mathfrak{n}\right)=0$. Thus $B\left(\left[\mathfrak{n}, \mathfrak{n}^{\perp}\right], \mathfrak{g}\right)=0$ and $0=\left[\mathfrak{n}, \mathfrak{n}^{\perp}\right]=\mathfrak{h}\left(\mathfrak{n}, \mathfrak{n}^{\perp}\right)$.

THEOREM 1. Let $\mathfrak{g}$ be a split simple Lie algebra. Let $\mathfrak{h}$ be a reductive subalgebra of $\mathfrak{g}$ which is normalized by a split Cartan subalgebra $\mathfrak{c}$ of $\mathfrak{g}(i . e ., \mathfrak{h}$ is reductive and regular [2]). Then $\mathfrak{h}$ has an $\operatorname{ad}(\mathfrak{c}+\mathfrak{h})$-stable complement $\mathfrak{m}$. Such an $\mathfrak{m}$ is either simple or abelian $\left(\mathfrak{m}^{2}=0\right)$.

Proof. We first show that $\mathfrak{c}+\mathfrak{h}$ is reductive. Letting $\mathfrak{g}=\mathfrak{g}_{0}+\sum \mathfrak{g}_{\alpha}$ be the root space decomposition of $\mathfrak{g}$, it suffices to show that for $\alpha \neq 0, \mathfrak{g}_{\alpha} \subseteq \mathfrak{c}+\mathfrak{h}$ implies $\mathfrak{g}_{-\alpha} \subseteq \mathfrak{c}+\mathfrak{h}[7$, p. 669]. Since $[\mathfrak{c}, \mathfrak{h}] \subseteq \mathfrak{h}$ we have $[\mathfrak{c}, \mathfrak{d}] \subseteq \mathfrak{d}$ where $\mathfrak{b}$ is the center of $\mathfrak{h}$. Thus $c+b$ is solvable. Thus $\operatorname{ad}(\mathfrak{c}+\delta)$ is triangulizable and $0=[\operatorname{ad} c, \operatorname{ad} \delta]=\operatorname{ad}[c, \delta]$

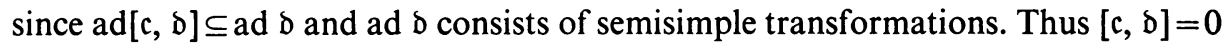
and $\mathfrak{b} \subseteq \mathfrak{c}=\mathfrak{g}_{0}$. Now $\mathfrak{h}=\mathfrak{b} \oplus \mathfrak{h}^{(1)}$ with $\mathfrak{h}^{(1)}$ semisimple, since $\mathfrak{h}$ is reductive. Let $\alpha$ be a nonzero root such that $\mathfrak{g}_{\alpha} \subseteq \mathfrak{c}+\mathfrak{h}$. Then since $\mathfrak{h}^{(1)}$ is ad $\mathfrak{c}$-stable and $\mathfrak{c}+\mathfrak{h}=\mathfrak{g}_{0}$ $+\mathfrak{d}+\mathfrak{h}^{(1)}=\mathfrak{g}_{0}+\mathfrak{h}^{(1)}$, we have $\mathfrak{g}_{\alpha} \subseteq \mathfrak{h}^{(1)}$. Now the restriction of the Killing form $B($,$) of g$ to $\mathfrak{h}^{(1)}$ is nondegenerate since it is the trace form of a faithful representation of the semisimple Lie algebra $\mathfrak{h}^{(1)}$ (see [3, p. 69]). Thus $B\left(\mathfrak{g}_{\alpha}, \mathfrak{h}^{(1)}\right) \neq 0$. Since $B\left(\mathfrak{g}_{\alpha}, \mathfrak{g}_{\beta}\right)=0$ for $\alpha+\beta \neq 0$, it follows $\mathfrak{g}_{-\alpha} \subseteq \mathfrak{h}^{(1)}$. Thus $\mathfrak{g}_{\alpha} \subseteq \mathfrak{c}+\mathfrak{h}$ implies $\mathfrak{g}_{-\alpha} \subseteq \mathfrak{c}+\mathfrak{h}$ and $\mathfrak{c}+\mathfrak{h}$ is reductive.

It follows that $\mathfrak{h}$ has a complement $\mathfrak{m}$ stable under $\operatorname{ad}(\mathfrak{c}+\mathfrak{h})$. Any complement $\mathfrak{m}$ is the sum of $\mathfrak{m} \cap \mathfrak{g}_{0}$ and those root spaces $\mathfrak{g}_{\beta}$ not occurring in $\mathfrak{h}$. In particular, $\mathfrak{g}_{\alpha} \subseteq \mathfrak{m}$ implies $\mathfrak{g}_{-\alpha} \subseteq \mathfrak{m}$.

We now show that such an $\mathfrak{m}$ is either simple or abelian. Assume that $\mathfrak{m}^{2} \neq 0$ and $\mathfrak{m}$ not simple. Then by Lemma $1, \mathfrak{m}$ has a proper Der $\mathfrak{m}$-stable ideal. Since $\mathfrak{m}$ is $\operatorname{ad}(\mathfrak{c}+\mathfrak{h})$-stable, $\operatorname{ad}(\mathfrak{c}+\mathfrak{h})$ consists of derivations of $\mathfrak{m}$. Thus $\mathfrak{m}$ has a proper ideal $\mathfrak{n}$ stable under $\operatorname{ad}(\mathfrak{c}+\mathfrak{h})$.

Let $\sigma$ be an automorphism of $\mathfrak{g}$ such that $\sigma \mid \mathfrak{c}=-i d_{\mathfrak{c}}$ and $\mathfrak{g}_{\alpha}^{\sigma}=\mathfrak{g}_{-\alpha}$ for all $\alpha$ (see $[3$, p. 127]). Then the above discussion shows that $\mathfrak{m}$ and $\mathfrak{h}$ are $\sigma$-stable. It follows that $(X Y)^{\sigma}=X^{\sigma} Y^{\sigma}$ and $(\mathfrak{h}(X, Y))^{\sigma}=\mathfrak{h}\left(X^{\sigma}, Y^{\sigma}\right)$. Thus $\sigma \mid \mathfrak{m}$ is an automorphism of $\mathfrak{m}$ and $\mathfrak{n}^{\sigma}$ is an ideal of $\mathfrak{m}$. Since $\left[\mathfrak{n}^{\sigma}, \mathfrak{c}+\mathfrak{h}\right]=\left[\mathfrak{n}^{\sigma},(\mathfrak{c}+\mathfrak{h})^{\sigma}\right]=[\mathfrak{n}, \mathfrak{c}+\mathfrak{h}]^{\sigma} \subseteq \mathfrak{n}^{\sigma}, \mathfrak{n}^{\sigma}$ is also $\operatorname{ad}(\mathfrak{c}+\mathfrak{h})$-stable.

Suppose that one of the ideals $\mathfrak{n} \cap \mathfrak{n}^{\sigma}, \mathfrak{n}+\mathfrak{n}^{\sigma}$ is proper in $\mathfrak{m}$. Call it $\mathfrak{p}$. Then $\mathfrak{p}$ is the sum of $\mathfrak{p} \cap \mathfrak{g}_{0}$ and root spaces $\mathfrak{g}_{\alpha}$. Moreover $\mathfrak{g}_{\alpha} \subseteq \mathfrak{p}$ implies $\mathfrak{g}_{-\alpha} \subseteq \mathfrak{p}$. It follows that $\mathfrak{m}=\mathfrak{m} \cap \mathfrak{g}_{0}+\mathfrak{p}+\mathfrak{p}^{\perp}$ where $\mathfrak{p}^{\perp}=\{X \in \mathfrak{m} \mid B(X, \mathfrak{p})=0\}$ (thus $\mathfrak{g}_{\alpha} \subseteq \mathfrak{m}-\mathfrak{g}_{0}$ and $\mathfrak{g}_{\alpha} \nsubseteq \mathfrak{p}$ implies $\mathfrak{g}_{-\alpha} \nsubseteq \mathfrak{p}$ which implies $B\left(\mathfrak{g}_{\alpha}, \mathfrak{p}\right)=0$ ). We use this to show that $\mathfrak{q}=\mathfrak{p}$ $+\mathfrak{h}(\mathfrak{p}, \mathfrak{p})$ is an ideal of $\mathfrak{g}$. By Lemma 2 it suffices to show that $\left[\mathfrak{p}, \mathfrak{p}^{\prime}\right] \subseteq \mathfrak{q}$ where $\mathfrak{p}^{\prime}=\mathfrak{p}^{\perp}+\mathfrak{m} \cap \mathfrak{g}_{0}$. But $\left[\mathfrak{p}, \mathfrak{m} \cap \mathfrak{g}_{0}\right] \subseteq[\mathfrak{p}, \mathfrak{c}] \subseteq \mathfrak{p}$. Thus it suffices to show that $\left[\mathfrak{p}, \mathfrak{p}^{\perp}\right]$ 
$\subseteq \mathfrak{q}$. But $B\left(\left[\mathfrak{p}, \mathfrak{p}^{\perp}\right], \mathfrak{c}+\mathfrak{h}\right)=B\left(\mathfrak{p}^{\perp},[\mathfrak{p}, \mathfrak{c}+\mathfrak{h}]\right)=B\left(\mathfrak{p}^{\perp}, \mathfrak{p}\right)=0$ and $\left[\mathfrak{p}, \mathfrak{p}^{\perp}\right] \subseteq(\mathfrak{c}+\mathfrak{h})^{\perp} \subseteq \mathfrak{m}$. Thus $\mathfrak{h}\left(\mathfrak{p}, \mathfrak{p}^{\perp}\right) \subseteq\left[\mathfrak{p}, \mathfrak{p}^{\perp}\right]+\mathfrak{p} \mathfrak{p}^{\perp} \subseteq \mathfrak{m}$ and $\mathfrak{h}\left(\mathfrak{p}, \mathfrak{p}^{\perp}\right)=0$. Thus $\left[\mathfrak{p}, \mathfrak{p}^{\perp}\right]=\mathfrak{p} \mathfrak{p}^{\perp} \subseteq \mathfrak{p} \subseteq \mathfrak{q}$ and $\mathfrak{q}$ is an ideal of $\mathfrak{g}$. Thus $\mathfrak{q}=\mathfrak{g}$ and $\mathfrak{n}$ cannot be proper in $\mathfrak{m}$, a contradiction.

Thus we have $\mathfrak{n} \cap \mathfrak{n}^{\sigma}=0$ and $\mathfrak{n}+\mathfrak{n}^{\sigma}=\mathfrak{m}$. Thus $\mathfrak{n} \cap \mathfrak{g}_{0}=\left(\mathfrak{n} \cap \mathfrak{g}_{0}\right)^{\sigma}=0$ (since $\left.\sigma \mid \mathfrak{g}_{0}=-\mathfrak{i d}_{\mathfrak{g}_{0}}\right)$. Thus $\mathfrak{m} \cap \mathfrak{g}_{0}=\mathfrak{n} \cap \mathfrak{g}_{0}+\left(\mathfrak{n} \cap \mathfrak{g}_{0}\right)^{\sigma}=0$. It follows that $B(\mathfrak{m}, \mathfrak{h})=0$ (e.g., $\mathfrak{m}=\sum_{\alpha \in S} \mathfrak{g}_{\alpha}$ for some set $S$ of nonzero roots, and $\alpha \in S$ implies $-\alpha \in S$ which implies $\mathfrak{g}_{-\alpha} \nsubseteq \mathfrak{h}$ and therefore $B\left(\mathfrak{g}_{\alpha}, \mathfrak{h}\right)=0$ ). Also $B(\mathfrak{n}, \mathfrak{n})=0$ (e.g., $\mathfrak{n}=\sum_{\alpha \in T} \mathfrak{g}_{\alpha}$ for some set $T$ of nonzero roots, and $\alpha \in T$ implies $-\alpha \notin T$ which implies $\left.B\left(\mathfrak{g}_{\alpha}, \mathfrak{n}\right)=0\right)$. It follows from Lemma 3 that $[\mathfrak{n}, \mathfrak{n}]=\mathfrak{n} \mathfrak{n}=\mathfrak{h}(\mathfrak{n}, \mathfrak{n})=0$. Thus $\mathfrak{n}^{\sigma} \mathfrak{n}^{\sigma}=0$. Finally $\mathfrak{m}^{2}=\left(\mathfrak{n}+\mathfrak{n}^{\sigma}\right)^{2}=\mathfrak{n}^{2}+\mathfrak{n} \mathfrak{n}^{\sigma}+\left(\mathfrak{n}^{\sigma}\right)^{2} \subseteq 0+\mathfrak{n} \cap \mathfrak{n}^{\sigma}+0=0$, a contradiction.

3. The semisimple case. We now consider the reductive pair $(\mathfrak{g}, \mathfrak{h})$ where $\mathfrak{g}$ is a simple Lie algebra and $\mathfrak{h}$ is a semisimple Lie subalgebra. We note that the Killing form $B($,$) of \mathfrak{g}$ restricted to $\mathfrak{h}$ is nondegenerate. For if $U, V \in \mathfrak{h}$, then $B(U, V)$ $=\operatorname{tr} \operatorname{ad}_{\mathfrak{g}} U \operatorname{ad}_{\mathfrak{g}} V$ is the trace form of the representation ad $\mathfrak{h}$ in $\mathfrak{g}$, and is nondegenerate by Cartan's criterion [3, p. 69]. (Note that $\operatorname{ad}_{\mathfrak{g}} U=0$ implies $U F$ is a one-dimensional ideal in the simple algebra $g$ so that $U=0$.) Thus if $\mathfrak{h}^{\perp}$ $=\{X \in \mathfrak{g} \mid B(X, \mathfrak{h})=0\}$, then $\mathfrak{h} \cap \mathfrak{h}^{\perp}=0$ and therefore $\mathfrak{g}=\mathfrak{h}^{\perp}+\mathfrak{h}$. And $B\left(\left[\mathfrak{h}^{\perp}, \mathfrak{h}\right], \mathfrak{h}\right)$ $=B\left(\mathfrak{h}^{\perp},[\mathfrak{h}, \mathfrak{h}]\right)=0$ so that for $\mathfrak{m}=\mathfrak{h}^{\perp},(\mathfrak{g}, \mathfrak{h})$ is a reductive pair with (fixed) decomposition $\mathfrak{g}=\mathfrak{m} \dot{+} \mathfrak{h}$. Note that since $\mathfrak{m}=\mathfrak{h}^{\perp}$, the Killing form $B$, restricted to $\mathfrak{m}$, is a nondegenerate invariant form, i.e., $B(X Y, Z)=B(X, Y Z)$.

THEOREM 2. Let $\mathfrak{g}$ be a simple Lie algebra and $\mathfrak{h}$ a semisimple subalgebra. Then $(\mathfrak{g}, \mathfrak{h})$ is a reductive pair with $\mathfrak{m}=\mathfrak{h}^{\perp}$. Furthermore $\mathfrak{m}^{2}=0$ or $\mathfrak{m}$ is simple.

Proof. Assume $\mathfrak{m}^{2} \neq 0$. Then we have from Lemma 1 that $\mathfrak{m}$ has a minimal proper ad $\mathfrak{h}$-stable ideal $\mathfrak{n}$. Then since $B$ is a nondegenerate invariant form on $\mathfrak{m}$ and $B([X U], Y)=B(X,[U Y])$ for $X, Y \in \mathfrak{m}, U \in \mathfrak{h}$, we have $\mathfrak{n}^{\perp}=\{X \in \mathfrak{m} \mid B(X, \mathfrak{n})$ $=0\}$ is an ad $\mathfrak{h}$-stable ideal of $\mathfrak{m}$. Thus $\mathfrak{n} \cap \mathfrak{n}^{\perp}$ is an ad $\mathfrak{h}$-stable ideal of $\mathfrak{m}$; and since $\mathfrak{n}$ is minimal, either $\mathfrak{n} \cap \mathfrak{n}^{\perp}=0$ or $\mathfrak{n} \cap \mathfrak{n}^{\perp}=\mathfrak{n}$.

In case $\mathfrak{n} \cap \mathfrak{n}^{\perp}=0$ we have $\mathfrak{m}=\mathfrak{n} \oplus \mathfrak{n}^{\perp}$. And we know from Lemma 3 that $\left[\mathfrak{n}, \mathfrak{n}^{\perp}\right]=0$. Thus $\mathfrak{q}=\mathfrak{n}+\mathfrak{h}(\mathfrak{n}, \mathfrak{n})$ is a proper ideal of $\mathfrak{g}$ by Lemma 2 . This contradiction shows we must have $\mathfrak{n} \cap \mathfrak{n}^{\perp}=\mathfrak{n}$.

In the case $\mathfrak{n} \cap \mathfrak{n}^{\perp}=\mathfrak{n}$ we can find an ad $\mathfrak{h}$-stable complement, $\mathfrak{n}^{\prime}$ (since ad $\mathfrak{h}$ is semisimple and therefore completely reducible); and we write $\mathfrak{m}=\mathfrak{n} \dot{+} \mathfrak{n}^{\prime}$. Thus since $B(\mathfrak{n}, \mathfrak{n})=0$, to show that $\mathfrak{n}=0$ it suffices to show $B\left(\mathfrak{n}, \mathfrak{n}^{\prime}\right)=0$.

To find a formula for $B(X, Y)$ with $X, Y \in \mathfrak{m}$, define $\varepsilon(X)$ and $\delta(X)$ by

$$
\begin{aligned}
\varepsilon(X): \mathfrak{m} \rightarrow \mathfrak{h}: & Y \rightarrow \mathfrak{h}(X, Y) \equiv \varepsilon(X)(Y), \\
\delta(X): \mathfrak{h} \rightarrow \mathfrak{m}: & U \rightarrow[X, U] \equiv \delta(X)(U),
\end{aligned}
$$

where $U \in \mathfrak{h}$. Using these maps we have for any $Z, X \in \mathfrak{m}, U \in \mathfrak{h}$ that

$$
\begin{aligned}
\left(\operatorname{ad}_{\mathfrak{g}} Z\right)(X) & =[Z, X]=Z X+\mathfrak{h}(Z, X) \\
& =(L(Z)+\varepsilon(Z))(X) \\
\left(\operatorname{ad}_{\mathfrak{g}} Z\right)(U) & =[Z, U]=\delta(Z)(U)
\end{aligned}
$$


and therefore

$$
\operatorname{ad}_{\mathfrak{g}} Z=\left(\begin{array}{cc}
L(Z) & \varepsilon(Z) \\
\delta(Z) & 0
\end{array}\right)
$$

From this, note that since $\mathfrak{g}$ is simple $0=\operatorname{tr} \operatorname{ad}_{\mathfrak{g}} Z=\operatorname{tr} L(Z)$. Also since $\mathfrak{h}=[\mathfrak{h}, \mathfrak{h}]$ is semisimple, and since $\mathfrak{h} \rightarrow \operatorname{ad}_{\mathfrak{m}} \mathfrak{h}: U \rightarrow \operatorname{ad}_{\mathfrak{m}} U$ and $\mathfrak{h} \rightarrow \operatorname{ad}_{\mathfrak{h}} \mathfrak{h}: U \rightarrow \operatorname{ad}_{\mathfrak{h}} U$ are representations of $\mathfrak{h}$, we have $\operatorname{tr} \operatorname{ad}_{\mathfrak{m}} U=\operatorname{tr} \operatorname{ad}_{\mathfrak{h}} U=0$ for all $U \in \mathfrak{h}$.

Next for $X, Y \in \mathfrak{m}$ define the linear transformation $\sigma(X, Y): \mathfrak{m} \rightarrow \mathfrak{m}$ by $\sigma(X, Y)$ $=\delta(X) \varepsilon(Y)$, that is, $\sigma(X, Y) Z=[X, \mathfrak{h}(Y, Z)](=[\mathfrak{h}(Z, Y), X])$. From (3) we have the identity

$$
\operatorname{ad}_{\mathfrak{m}} \mathfrak{h}(X, Y)-\sigma(X, Y)+\sigma(Y, X)=[L(X), L(Y)]-L(X Y)
$$

and therefore $\operatorname{tr} \sigma(X, Y)=\operatorname{tr} \sigma(Y, X)$. From this and the matrix for $\operatorname{ad}_{\mathfrak{g}} Z$ we obtain for $X, Y \in \mathfrak{m}$ that

$$
\begin{aligned}
B(X, Y) & =\operatorname{tr} \operatorname{ad}_{\mathfrak{g}} X \operatorname{ad}_{\mathfrak{g}} Y \\
& =\operatorname{tr} L(X) L(Y)+\operatorname{tr} \varepsilon(X) \delta(Y)+\operatorname{tr} \delta(X) \varepsilon(Y) \\
& =\operatorname{tr} L(X) L(Y)+\operatorname{tr} \delta(Y) \varepsilon(X)+\operatorname{tr} \delta(X) \varepsilon(Y) \\
& =\operatorname{tr} L(X) L(Y)+\operatorname{tr} \sigma(Y, X)+\operatorname{tr} \sigma(X, Y) \\
& =\operatorname{tr} L(X) L(Y)+2 \operatorname{tr} \sigma(X, Y),
\end{aligned}
$$

using for the third equality that if $S \in \operatorname{Hom}(V, W)$ and $T \in \operatorname{Hom}(W, V)$ for vector spaces $V$ and $W$, then $\operatorname{tr} S T=\operatorname{tr} T S$.

Now recall that in the decomposition $\mathfrak{m}=\mathfrak{n}+\mathfrak{n}^{\prime}$ we must show $B\left(\mathfrak{n}, \mathfrak{n}^{\prime}\right)=0$. Thus for $X \in \mathfrak{n}, Y \in \mathfrak{n}^{\prime}$ we.have (from the fact that $\mathfrak{n}$ is an ideal and $\mathfrak{n n}=0$ ) the matrices

$$
L(X)=\left(\begin{array}{cc}
0 & 0 \\
X_{21} & 0
\end{array}\right) \text { and } L(Y)=\left(\begin{array}{cc}
Y_{11} & 0 \\
Y_{21} & Y_{22}
\end{array}\right)
$$

and therefore $\operatorname{tr} L(X) L(Y)=0$ and $B(X, Y)=2 \operatorname{tr} \sigma(X, Y)$.

To find the matrix for $\sigma(X, Y)$ (with $\left.X \in \mathfrak{n}, Y \in \mathfrak{n}^{\prime}\right)$ let $Z \in \mathfrak{n}, Z^{\prime} \in \mathfrak{n}^{\prime}$. Then

Therefore

$$
\begin{aligned}
\sigma(X, Y) Z & =[\mathfrak{h}(Z, Y), X] \in \mathfrak{n}, \\
\sigma(X, Y) Z^{\prime} & =\left[\mathfrak{h}\left(Z^{\prime}, Y\right), X\right] \in \mathfrak{n} .
\end{aligned}
$$

$$
\sigma(X, Y)=\left(\begin{array}{ll}
\sigma_{11} & 0 \\
\sigma_{21} & 0
\end{array}\right)
$$

and $\operatorname{tr} \sigma(X, Y)=\operatorname{tr} \sigma_{11}=\operatorname{tr}_{\mathfrak{n}} \sigma(X, Y)$. To find the action of $\sigma(X, Y)$ on $\mathfrak{n}$ again let $Z \in \mathfrak{n}$. Then since $\mathfrak{n}$ is an ideal, $\mathfrak{n} \mathfrak{n}=0$ and $\mathfrak{h}(\mathfrak{n}, \mathfrak{n})=0$, we have from (3) that

$$
\begin{aligned}
0=J(Z, X, Y) & =[Z, \mathfrak{h}(X, Y)]+[X, \mathfrak{h}(Y, Z)] \\
& =\left[-\operatorname{ad}_{\mathfrak{n}} \mathfrak{h}(X, Y)+\sigma(X, Y)\right] Z .
\end{aligned}
$$

Therefore on $\mathfrak{n}$ we have $\sigma(X, Y)=\operatorname{ad}_{\mathfrak{n}} \mathfrak{h}(X, Y)$ and since $U \rightarrow \operatorname{ad}_{\mathfrak{n}} U$ is a representation of the semisimple Lie algebra $\mathfrak{h}, 0=\operatorname{tr}_{\mathfrak{n}} \mathfrak{h}(X, Y)=\operatorname{tr}_{\mathfrak{n}} \sigma(X, Y)$. Thus $B\left(\mathfrak{n}, \mathfrak{n}^{\prime}\right)=0$ and $\mathfrak{m}$ is simple, a contradiction. Thus either $\mathfrak{m}^{2}=0$ or $\mathfrak{m}$ is simple. 
4. Remarks. (i) The above discussion for $\mathfrak{h}$ semisimple holds for $\mathfrak{h}$ reductive in $\mathfrak{g}$ except for the assertion that $\operatorname{tr} \operatorname{ad}_{\mathfrak{n}} \mathfrak{h}(X, Y)=0$ and its consequences. The authors do not know whether the theorem holds for all reductive $\mathfrak{h}$.

(ii) If $\mathfrak{h}$ is the zero-space of a derivation of $\mathfrak{g}$ or the one-space of an automorphism of $\mathfrak{g}$, then $\mathfrak{h}$ is reductive and contains a regular element of $\mathfrak{g}$ [1]. Thus if $\mathfrak{g}$ is simple and the underlying field algebraically closed, the associated $\mathfrak{m}$ is simple or abelian by Theorem 1 .

(iii) It would be of value to determine all pairs $(\mathfrak{g}, \mathfrak{h})$ with $\mathfrak{g}$ semisimple for which an associated $\mathfrak{m}$ is simple. We now give an example of one nontrivial such pair $(\mathfrak{g}, \mathfrak{h})$ where $\mathfrak{g}$ is not simple. Thus let $\mathfrak{g}=\mathfrak{g}_{1} \oplus \mathfrak{g}_{2}$ (direct) where the $\mathfrak{g}_{i}(i=1,2)$ are real compact simple Lie algebras. Suppose that $\mathfrak{b}$ is a simple subalgebra of $\mathfrak{g}_{1}$, $\mathfrak{b}^{\prime}$ a simple subalgebra of $\mathfrak{g}_{2}, B \rightarrow B^{\prime}$ an isomorphism from $\mathfrak{b}$ onto $\mathfrak{b}^{\prime}$. Let $\mathfrak{h}$ $=\left\{B+B^{\prime} \mid B \in \mathfrak{b}\right\}$ and $m=\mathfrak{h}^{\perp}$. Then $\mathfrak{g}_{1}, \mathfrak{g}_{2}, \mathfrak{b}$, and $\mathfrak{b}^{\prime}$ can easily be chosen such that $\mathfrak{m}^{2} \neq 0$. We claim that for any such choice, $\mathfrak{m}$ is simple. By Lemma 1 , it suffices to show that $\mathfrak{m}$ has no proper ad $\mathfrak{h}$-stable ideal. If $\mathfrak{n}$ were such an ideal, then since the Killing form is negative definite on $\mathfrak{g}, \mathfrak{m}=\mathfrak{n} \oplus \mathfrak{n}^{\perp}$. It is now clear that $\mathfrak{n}+\mathfrak{h}(\mathfrak{n}, \mathfrak{n})$ is an ideal of $\mathfrak{g}$ by Lemma 2 , since $\left[\mathfrak{n}, \mathfrak{n}^{\perp}\right]=0$ by Lemma 3. But then $\mathfrak{n}+\mathfrak{h}(\mathfrak{n}, \mathfrak{n})=\mathfrak{g}_{1}$ or $\mathfrak{g}_{2}$. But by construction, $\mathfrak{h} \cap \mathfrak{g}_{1}=\mathfrak{h} \cap \mathfrak{g}_{2}=0$. Thus $\mathfrak{n}=\mathfrak{g}_{1}$ or $\mathfrak{g}_{2}$. This is impossible since $B(\mathfrak{n}, \mathfrak{h})=0$ whereas $B\left(\mathfrak{g}_{i}, \mathfrak{h}\right) \neq 0$ for $i=1,2$.

\section{BIBLIOGRAPHY}

1. A. Borel and G. D. Mostow, On semisimple automorphisms of Lie algebras, Ann. of Math. 61 (1955), 389-504.

2. E. B. Dynkin, Semisimple subalgebras of semisimple Lie algebras, Amer. Math. Soc. Transl. (2) 6 (1957), 111-244.

3. N. Jacobson, Lie algebras, Wiley, New York, 1962.

4. B. Kostant, On differential geometry and homogeneous spaces. I, II, Proc. Nat. Acad. Sci. U.S.A. 42 (1956), 258-261; 354-357.

5. K. Nomizu, Invariant affine connections on homogeneous spaces, Amer. Math. J. 76 (1954), 33-65.

6. A. Sagle, On anti-commutative algebras and homogeneous spaces, J. Math. Mech. (to appear).

7. A. Borel and J. Tits, Groupes réductifs, pp. 659-755, Inst. Hautes Etudes Sci. Paris No. 27, 1965.

UNIVERSITY OF MINNESOTA,

MinNeapolis, Minnesota

YALE UNIVERSITY,

New Haven, Connecticut 